Results Synergies and gaps in the availability of data and in policies and programming were identified across EU/EEA. Eleven countries ever adopted a national sexual health strategy and 24 implemented a national HIV/STI prevention strategy. Specific HIV/ STI prevention strategies targeting vulnerable groups were identified for: young people (24 countries), MSM (22 countries), sex workers (19 countries), migrants (20 countries) and injecting drug users (24 countries). National surveys on sexual knowledge, attitude and practise were conducted in general population by 19 countries. Eighteen countries collect data on sexual health in MSM, 9 in PLHIV and 6 in migrants. Sexuality education is mandatory in 20 countries. In the last 5 years, 24 Member States conducted HIV/ AIDS awareness campaigns.

Conclusions The following mechanisms are considered to support Member States and to promote a comprehensive approach for HIV/STI prevention in the context of sexual health: endorse synergy between European bodies and NGOs in regards to data collection, reporting and publication; development of a standardised set of indicators for collecting comparable data; development of an evidence-based framework to allow countries to evaluate their sexual health interventions; facilitate communication among (inter)national experts through a dedicated sexual health platform.

\section{SO3.5 ADVANCING SEXUAL HEALTH IN THE US: FROM THEORY TO ACTION}

doi:10.1136/sextrans-2013-051184.0024

J M Douglas. National Center for HIVIAIDS, Viral Hepatitis, STD, and TB Prevention, Centers for Disease Control and Prevention, GA, United States

Background The United States faces a variety of public health issues related to sexual behaviour including HIV, STD, unintended pregnancy, and sexual violence. A 2010 CDC consultation on A Public Health Approach for Advancing Sexual Health in the United States (http:// www.cdc.gov/sexualhealth/docs/SexualHealthReport-2011-508. pdf) provided recommendations on how CDC could enhance prevention programme impact by comprehensively addressing sexual health.

Methods Since the consultation, CDC has worked across its divisions and with external partners to translate the theoretical concept of sexual health into public health action through national leadership, partnership development, and scientific and programme efforts. Results Initially, we developed a sexual health framework emphasising the importance of using sexual health promotion to complement and enhance core disease control and prevention activities based on five key principles: acknowledgment of the impact of sexual health on overall health, consideration of underlying social issues, an emphasis on wellness, a focus on relationships, and integrated prevention approaches. Subsequently, we reviewed U.S. national data systems to identify existing measures of key domains of sexual health and conducted health communications research assessing optimal frameworks and messages for communicating with audiences with varied perspectives. Partnership development was accelerated by forming a National Sexual Health Coalition, with a spectrum of non-governmental partners addressing sexual health among the general population, adolescents, and MSM. Public health implementation of sexual health promotion has focused on supporting development of sexual health plans by U.S. states and sexual health training for providers in public health and primary care settings.

Conclusions Implementing actions to address sexual health in a society with diverse values is a challenging and evolving process, but has great potential to improve prevention efforts by engaging a spectrum of partners, facilitating societal dialogue around sexuality and sexual behaviour, reducing stigma and fear, and enhancing efficiency and effectiveness of prevention messages and services.

\section{S.04 - Scaling up of HIV treatment programmes among the most at-risk populations in low- and middle-income countries}

\section{S04.1 SCALING UP OF HIV TREATMENT PROGRAMMES AMONG THE MOST AT-RISK POPULATIONS IN LOW- AND MIDDLE- INCOME COUNTRIES - INTRODUCTION}

doi:10.1136/sextrans-2013-051184.0025

1,2,3 M Alary. 'CHU de Québec, Québec, OC, Canada; '2Université Laval, Québec, OC, Canada; 'Institut national de santé publique du Québec, Québec, OC, Canada

Although access to antiretroviral therapy (ART) for HIV infection in low- and middle-income countries has tremendously improved over the last decade, it remains far from optimal in most settings when it comes to the treatment of marginalised populations, such as sex workers, injection drug users and men who have sex with men. Small-scale demonstration projects indicate that ART can be successfully implemented among sex workers, with satisfactory immunological and virological responses, good treatment compliance levels, and maintenance or even improvement of safe sex behaviour. Some of the studies carried out so far underline the importance of strongly linking prevention with care through the integration of HIV/STI care services and community-based prevention packages. Scaling-up treatment programmes for marginalised, hard-to-reach populations, will undoubtedly be challenging. Nevertheless, in addition to the health equity issues related to access to ART for such populations, the application of targeted "test and treat" strategies could substantially impact HIV prevention at the general population level, given recent trial data showing that the implementation of this strategy among sero-discordant couples led to a $96 \%$ reduction in HIV transmission. Such a strategy should however be carefully evaluated in the target population before implementation and scale-up, and would require specific adherence support and community involvement.

It is in this context that this symposium will present practical experiences in implementing ART programmes among female sex workers in the context of generalised, concentrated and mixed epidemics. Modelling studies of the preventive impact of such programmes across different epidemic contexts, and with different criteria for ART initiation (including "test and treat"), will also be presented. A similar study about injection drug users in the context of a concentrated epidemic will complete the presentations in this symposium that should stimulate a fruitful discussion period between the participants and the speakers.

\section{S04.2 MODELLING THE IMPACT OF EARLY ANTI-RETROVIRAL TREATMENT AMONGST PEOPLE WHO INJECT DRUGS IN A HIGH PREVALENCE SETTING}

doi:10.1136/sextrans-2013-051184.0026

\section{P Vickerman. LSHTM, London, UK}

Background HIV transmission in many settings is concentrated amongst people who inject drugs (PWID), and can reach high HIV prevalence. Early anti-retroviral treatment (ART) for PWID could be important for reducing HIV transmission in these settings. This modelling analysis projects the impact of such a strategy in a high HIV prevalence (30\%) setting, and explores how behavioural and epidemiological factors affect the projections.

Methods A deterministic, model of HIV transmission amongst PWID is calibrated to HIV prevalence data from Manipur, India. 